\title{
Evaluation of serum ATX and LPA as potential diagnostic biomarkers in patients with pancreatic cancer
}

\author{
Jiang Chen, Hongyu Li, Wenda Xu and Xiaozhong Guo* (1)
}

\begin{abstract}
Background: Pancreatic cancer (PC) is a devastating disease that has a poor prognosis and a total 5 -year survival rate of around 5\%. The poor prognosis of PC is due in part to a lack of suitable biomarkers that can allow early diagnosis. The lysophospholipase autotaxin (ATX) and its product lysophosphatidic acid (LPA) play an essential role in disease progression in PC patients and are associated with increased morbidity in several types of cancer. In this study, we evaluated both the potential role of serum LPA and ATX as diagnostic markers in PC and their prognostic value for PC either alone or in combination with CA19-9.
\end{abstract}

Methods: ATX, LPA and CA19-9 levels were evaluated using ELISA of serum obtained from PC patients $(n=114)$ healthy volunteers (HVs: $n=120)$ and patients with benign pancreatic diseases (BPDs: $n=94)$.

Results: Serum levels of ATX, LPA and CA19-9 in PC patients were substantially higher than that for BPD patients or HVs $(p<0.001)$. The sensitivity of LPA in early phase PC was $91.74 \%$ and the specificity of ATX was $80 \%$. The levels of ATX, LPA and CA19-9 were all substantially higher for early stage PC patients compared to levels in serum from BPD patients and HVs. The diagnostic efficacy of CA19-9 for PC was significantly enhanced by the addition of ATX and LPA $(p=0.0012)$.

Conclusion: Measurement of LPA and ATX levels together with CA19-9 levels can be used for early detection of PC and diagnosis of $\mathrm{PC}$ in general.

Keywords: Biological markers, Diagnosis, Lysophospholipids, Autotaxin, Pancreatic neoplasms

\section{Background}

Early diagnosis and improved treatments have together contributed to increased survival rates for several types of cancer over the recent decades. However, pancreatic cancer (PC) continues to be one of the deadliest cancers and its prognosis remains poor [1]. In patients with resect-able tumors, the 5-year survival rate for $\mathrm{PC}$ ranges from 15 to $20 \%$. For those PC patients presenting with unresectable tumors and distant metastases, the 5-year

\footnotetext{
*Correspondence: guoxiaozhong1962@126.com

Department of Gastroenterology, General Hospital of Northern Theater Command, No. 83 Wenhua Road, Shenyang 110840, Liaoning Province,
} China survival rate is nearly $0 \%$ [2]. This poor prognosis and low survival rate are largely due to a lack of recognizable symptoms and early detection markers that result in delays in diagnosis [3]. Thus, there is an urgent need for new biomarkers that can allow early detection of PC and reduce the likelihood of metastasis.

CA19-9 is regarded as a gold standard marker that is applied for diagnosis (screening and staging) and monitoring of PC progression (measure of resectability, early identification of recurrence, and prediction of therapeutic response) [4]. However, as a biomarker, CA19-9 has several limitations for diagnosing early stage PC including low expression levels and specificity as well as 
inconsistent presentation in PC patients [5]. Therefore, new, more sensitive biomarkers that can be used either alone or in combination with previously defined biomarkers are needed to increase the likelihood of early PC detection [6].

Increasing evidence now supports a physiological role for lysophosphatidic acid (LPA) in regulating tumor progression, angiogenesis, and metastasis. LPA is a bioactive phospholipid that engages at least six receptors, LPAR1-6, which are each coupled to a distinct G-protein [7] that participates in various cellular activities such as cell migration, proliferation, and differentiation [8]. LPA also contributes to progression, metastasis, and initiation of various cancers, including PC [9]. LPA is present in various biological fluids, and its levels in plasma are well-characterized in terms of its role in blood coagulation. LPA is generated from lysophospholipase D (lysoPLD)-mediated hydrolysis of lysophosphatidylcholine (LPC) [10]. Total plasma LPA is considered to be a biomarker for diagnosis of gastric tumors [11].

Autotaxin (ATX), also referred to as ectonucleotide pyrophosphatase / phosphodiesterase 2 (ENPP2), is a $125 \mathrm{kDa}$ secreted glycoprotein that was originally discovered as an autocrine cell motility factor produced by melanoma cells cultured in conditioned medium [12]. ATX was primarily thought to act as a pyrophosphatase [13], but subsequent studies showed that ATX could hydrolyze LPC to form LPA [14]. Augmented ATX expression has been reported in breast cancer, $\mathrm{PC}$, hematological cancer, and thyroid and hepatocellular carcinoma [15]. ATX levels can be correlated with cancer cell invasiveness $[16$, 17].

Ex vivo and in vivo studies showed that elevated ATXLPA signaling activity can contribute to cancer progression and initiation [18].]. However, whether plasma levels of ATX and LPA could serve as diagnostic biomarkers for $\mathrm{PC}$ is unclear. In this study, we evaluated the potential of plasma LPA and ATX as cancer biomarkers and assessed whether they could be used alone or in combination with CA19-9 to allow early detection of PC.

\section{Methods}

\section{Patients}

Serum samples were gathered from 328 individuals, who were divided into three groups: Group A had 114 PC patients, Groups B and Group C served as cohort control groups and comprised 120 healthy volunteers (HVs) and 94 patients with benign pancreatic diseases (BPDs), respectively. All the patients were consecutively admitted to the General Hospital of Northern Theater Command, Shenyang, China between January 2013 and May 2019. The research protocol was adopted through the Ethics Committee of the General Hospital of Northern Theater
Command, and the number was $\mathrm{K}(2012) 49$. Informed written consents were obtained for all participants.

"High sugar diet" was defined according to the standard of the American Heart Association (AHA) [19]. For men, Eating more than $37.5 \mathrm{~g}$ or 9 teaspoons of sugar a day is considered a high sugar diet. For women, the standard was changed to $25 \mathrm{~g}$ or 6 teaspoons of sugar per day. The "Alcohol consumption" was defined according to the standard of the National Institute on Alcohol Abuse and Alcoholism (NIAAA) [20]. The excessive drinking is $14 \times 8 \mathrm{~g}$ pure alcohol / day or $14 \times 20 \mathrm{~g}$ pure alcohol / week for men, and $14 \times 4 \mathrm{~g}$ pure alcohol / day or $14 \times 8 \mathrm{~g}$ pure alcohol / week for women. In this study, High sugar diet and alcohol consumption were ascertained at all visits by means of an interviewer-administered dietary questionnaire. In calculating the amount of sugar or alcohol consumed (in grams per day or per week), people were judged whether they currently with the high sugar diet or excessive drinking.

CT scan and chest X-ray, as well as biological and physical examinations, were conducted during pre-treatment investigations. All patients in all groups had regular blood chemistry and full clinical tests. Imaging, blood chemistry and physical test results for the HVs were within normal ranges. Patients with PC were diagnosed based on histopathological or clinical observations (history of pancreatic disease, clinical presentation, laboratory tests and abdominal imaging). None had undergone radio- or chemotherapy prior to serum collection. Diagnosis of BPDs was based on the absence of malignant features on imaging studies for a one year follow-up.

\section{Samples}

A total of $10 \mathrm{~mL}$ of venous blood was collected from each participant into sterile vacutainers. The samples were centrifuged at 3,500 $\mathrm{g}$ for $10 \mathrm{~min}$ and serum aliquots were stored at $-80{ }^{\circ} \mathrm{C}$. The samples were thawed prior to measurement of ATX, LPA and CA19-9 blood levels. Blood biochemical parameters including total bilirubin, albumin, alanine alkaline phosphatase, aspartate aminotransferase, $\gamma$-glutamyl transferase, transaminase, glucose, creatinine, prothrombin time, D-dimer and activated partial prothrombin time were measured using a Siemens ADVIA1800 chemistry analyzer.

Serum levels of LPA, ATX and CA19-9 were assayed by ELISA (Cloud-Clone Corp., TX, USA) and estimated by interpolation from a standard curve produced using known amounts of each protein. The samples were serially diluted with PBS and assessed again to ensure that the concentrations were within measurable ranges. The investigators were blinded to sample origin and all tests were executed in triplicate following the manufacturer's instructions. 


\section{Statistical analysis}

All statistical analyses were executed using the SPSS v20.0 package. Uninterrupted data are shown as median with range and mean \pm standard error of the mean (SEM). An independent sample $t$-test was used to compared continuous data. Differences among categories were examined using the Mann-Whitney $U$ test. To describe the prognostic precision performance of the three markers as diagnostic biomarkers, receiver operating characteristics (ROC) analysis was used. The AUC was calculated and compared by the De Long test. Reversible $p<0.05$ was regarded as statistically significant.

\section{Results}

Characteristics of PC and BPD patients and healthy volunteers

Overall, $120 \mathrm{HVs}$, 94 patients with BPDs and 114 PC patients were enrolled in this study. The patient demographics are summarized in Table 1. Of the 114 PC patients, $36(31.6 \%)$, and $78(68.4 \%)$ were female and male, respectively. The age of this group ranged from 40

Table 1 Characteristics of patients and healthy volunteers

\begin{tabular}{|c|c|c|c|c|c|c|c|c|}
\hline \multirow[t]{2}{*}{ Characteristics } & \multicolumn{2}{|c|}{$\begin{array}{l}\text { PC patients } \\
(n=114)\end{array}$} & \multicolumn{2}{|c|}{$\begin{array}{l}\text { Patients with BPDs } \\
(n=94)\end{array}$} & \multicolumn{2}{|c|}{$\begin{array}{l}\text { HVs } \\
(n=120)\end{array}$} & \multirow[t]{2}{*}{$X^{2}$} & \multirow[t]{2}{*}{$p$ value } \\
\hline & No & $\%$ & No & $\%$ & No & $\%$ & & \\
\hline \multicolumn{9}{|l|}{ Age (years) } \\
\hline $\begin{array}{l}\text { Median } \\
\text { (range) }\end{array}$ & $\begin{array}{l}59 \\
(40-82)\end{array}$ & & $\begin{array}{l}56 \\
(31-83)\end{array}$ & & $\begin{array}{l}60 \\
(37-84)\end{array}$ & & & 0.642 \\
\hline \multicolumn{9}{|l|}{ Sex } \\
\hline Female & 36 & 31.6 & 27 & 28.7 & 36 & 30 & 6.183 & 0.792 \\
\hline Male & 78 & 68.4 & 67 & 71.3 & 84 & 70 & 0.169 & 0.572 \\
\hline High sugar diet & 54 & 47.3 & 15 & 16.1 & 24 & 20 & 4.097 & $0.043^{*}$ \\
\hline Smoker & 60 & 52.6 & 11 & 11.7 & 26 & 21.7 & 5.653 & $0.017^{*}$ \\
\hline Alcohol consumption & 6 & 5.26 & 5 & 5.3 & 6 & 5 & 0.002 & 0.966 \\
\hline Family history of PC & 15 & 13.2 & 7 & 7.4 & 6 & 5 & 0.924 & 0.336 \\
\hline \multicolumn{9}{|l|}{ Location } \\
\hline Head & 81 & 71.1 & & & & & & \\
\hline Body/rear & 33 & 28.9 & & & & & & \\
\hline \multicolumn{9}{|l|}{ Histologic grade } \\
\hline I & 12 & 10.5 & & & & & & \\
\hline$\|$ & 84 & 73.7 & & & & & & \\
\hline$|-| \mid$ & 18 & 15.8 & & & & & & \\
\hline \multicolumn{9}{|l|}{ Tumor size $(\mathrm{cm})$} \\
\hline Median & 3.94 & & & & & & & \\
\hline Range & $1-6$ & & & & & & & \\
\hline \multicolumn{9}{|l|}{ LNM } \\
\hline Positive & 42 & 36.8 & & & & & & \\
\hline Negative & 54 & 47.3 & & & & & & \\
\hline Unknown & 18 & 15.9 & & & & & & \\
\hline \multicolumn{9}{|l|}{$\mathrm{PNI}$} \\
\hline Positive & 109 & 95.6 & & & & & & \\
\hline Negative & 5 & 4.4 & & & & & & \\
\hline \multicolumn{9}{|l|}{ Tumor stages (TS) } \\
\hline $1 b$ & 9 & 7.8 & & & & & & \\
\hline $2 a$ & 21 & 18.4 & & & & & & \\
\hline $2 b$ & 15 & 13.1 & & & & & & \\
\hline 3 & 18 & 15.8 & & & & & & \\
\hline 4 & 51 & 44.9 & & & & & & \\
\hline
\end{tabular}

BPDs, benign pancreatic diseases; LNM, lymph node metastasis; PNI, perineural invasion; HVs, healthy volunteers

* Depicts results were significant at $p<0.05$ 
to 82 years-old, and the median age was 59 years-old. The median tumor size was $3.94 \mathrm{~cm}$ (range from 1.0$6.2 \mathrm{~cm}$ ). For the majority of PC patients (81 cases, 71.1\%), the tumor was located in the head of the pancreas, followed by the tail or body (33 cases, 28.9\%). Regional lymph node metastasis (LNM) was present in 14 (36.8\%) PC patients, absent in 18 patients (47.3\%) and 2 patients (5.2\%) had duodenum metastasis. Pancreatic ductal adenocarcinoma was diagnosed for 107 patients whereas 7 patients had pancreatic acinar cell carcinoma. Pathologic grade I was seen for 12 patients (10.5\%), whereas grade II and grade I to II was seen for 84 (73.7\%) and 18 (15.8\%) patients, respectively. Perineural invasion (PNI) was observed for 109 cases $(95.6 \%)$. In terms of tumor stage, 9 (7.7\%), 35 (31.6\%), 18 (15.8\%), and 51 (44.9\%) cases were Stage 1, 2, 3, and 4, respectively. Autoimmune pancreatitis ( $\mathrm{n}=8,5$ males and 3 females), chronic pancreatitis $(\mathrm{n}=62,51$ males and 11 females $)$ and pancreatic cyst disease ( $n=24,17$ males and 7 females) were represented among the 94 BPD patients.

Compared with the cohort control groups, the PC patient group had a significantly higher proportion of smokers and those who consumed a diet high in sugar $(p<0.05)$. Age, sex, presence of chronic pancreatitis, alcohol consumption, and family history of PC were statistically similar among all three groups.

\section{Biochemical parameters for healthy volunteers, PC patients, and BPD patients}

Results for analysis of blood samples obtained from 120 HVs and 94 BPD patients were analyzed by comparison with $114 \mathrm{PC}$ patients. The PC patients had not undergone radio- or chemotherapy prior to the blood collection. PC patients had alanine transaminase, aspartate aminotransferase, alkaline phosphatase, and $\gamma$-glutamyl transferase activity that was 2.3-, 2.8-, 1.5-, and 2.3-fold higher than that for the HVs $(p<0.05)$. Meanwhile, the total serum level of bilirubin, creatinine, and D-dimer content in PC patients was 6.2-, 1.3-, and 3.8-fold higher than that for HVs $(p<0.001)$. Compared with the HVs, the blood glucose level of PC patients was 1.8 -fold higher $(p<0.05)$ and the activated partial prothrombin time expressed as prothrombin time was 1.4 -fold longer $(p<0.05)$. The albumin content in PC patients was lower $(p<0.05)$ compared with that in HVs (Table 2).

Comparing PC patients with BPD patients, PC patients had alanine transaminase, aspartate aminotransferase, alkaline phosphatase, and $\gamma$-glutamyl transferase activities that were increased by 1.75-, 2.05-, 1.36-, and 1.9-fold $(p<0.05)$ more than that for BPD patients. The total content of bilirubin, creatinine, and D-dimer contents in PC patients was 4.9-, 1.2-, and 2.6-fold higher than that for BPD patients $(p<0.001)$. PC and BPD patients had similar blood glucose levels and activated partial prothrombin time $(p>0.05)$. The albumin content in PC patients was depressed $(p<0.001)$ compared to that for BPD patients (Table 2).

Comparison of ATX, LPA, and CA19-9 serum concentrations for HVs, PC patients, and BPD patients

The plasma levels of ATX, LPA and CA19-9 in PC patients were significantly higher than that for the HV group ( $p<0.001)$ and the BPD group $(p<0.001)$ (Table 3$)$. Upon considering tumor stage, ATX, LPA and CA19-9

Table 2 Comparison of biochemical parameters among PC patients, patients with benign pancreatic diseases and healthy volunteers

\begin{tabular}{|c|c|c|c|c|c|}
\hline \multirow[t]{2}{*}{ Variables } & \multirow{2}{*}{$\begin{array}{l}\text { PC patients } \\
(n=114) \\
\text { Median (range) }\end{array}$} & \multirow{2}{*}{$\begin{array}{l}\text { Patients with BPDs } \\
(\mathrm{n}=94) \\
\text { Median (range) }\end{array}$} & \multirow{2}{*}{$\begin{array}{l}\text { HVs } \\
(n=120) \\
\text { Median (range) }\end{array}$} & \multicolumn{2}{|l|}{$p$ value (s) } \\
\hline & & & & PC vs. BPDs & PC vs. HVs \\
\hline Total bilirubin, $\mu \mathrm{mol} / \mathrm{L}$ & $44.8(19.2-141.5)$ & $9.16(6.3-72.8)$ & $7.24(3.68-15.1)$ & $<0.001$ & $<0.001$ \\
\hline Albumin, $g / L$ & $31.48(20.1-83.5)$ & $42(39.7-65.4)$ & $51.3(27.1-168.3)$ & $<0.001$ & $<0.001$ \\
\hline Alanine transaminase, $\mathrm{U} / \mathrm{L}$ & $42(34.1-144)$ & $24.3(24.3-59.5)$ & $18.8(13.3-41)$ & $<0.001$ & $<0.001$ \\
\hline Aspartate aminotransferase, U/L & $116.9(62-328.4)$ & $37.5(27.9-212.4)$ & $27.2(18-59.6)$ & $<0.001$ & $<0.001$ \\
\hline Alkaline phosphatase, U/L & $109.7(39.2-247.6)$ & $80.4(19.3-169.2)$ & $71.2(41.5-145.2)$ & $0.008^{*}$ & $0.005^{*}$ \\
\hline ү-glutamyl transferase, U/L & $102.3(77.5-201.2)$ & $51.4(25-110.2)$ & $44.7(37.2-88.6)$ & $0.005^{*}$ & $0.004^{*}$ \\
\hline Glucose, mmol/L & $9.3(8.8-28.7)$ & $8.7(3.4-42.6)$ & $4.5(4.1-8.2)$ & 0.086 & $<0.001$ \\
\hline Creatinine, $\mu \mathrm{mol} / \mathrm{L}$ & $87.3(45.3-143.3)$ & $71.2(19.3-95.5)$ & $64.7(32.6-213.8)$ & $0.043^{*}$ & $0.027^{*}$ \\
\hline Prothrombin time, sec & $17.3(4.5-56.1)$ & $18.4(8.7-92.6)$ & $12.6(10.6-58)$ & 0.074 & $0.018^{*}$ \\
\hline Activated partial prothrombin time, sec & $44.5(37.4-103.5)$ & $27.3(10.9-90.1)$ & $30.5(22.2-67.5)$ & $<0.001$ & $<0.001$ \\
\hline D-dimer, $\mu \mathrm{g} / \mathrm{mL}$ & $0.8(0.4-2.3)$ & $0.3(0.08-1.9)$ & $0.2(0.03-1.2)$ & $<0.001$ & $<0.001$ \\
\hline
\end{tabular}

BPDs, benign pancreatic diseases; $\mathrm{HVs}$, healthy volunteers

* Depicts results were significant at $p<0.05$ 


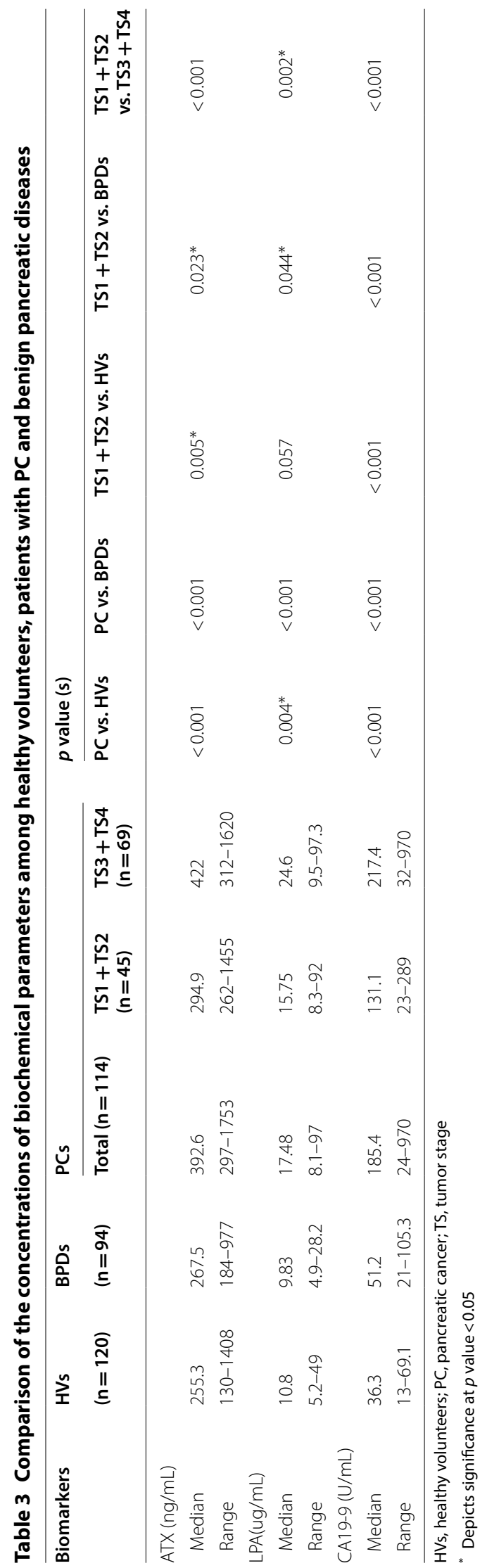


levels for TS1 \& TS2 patients were all significantly higher ( $p=0.023, p=0.044$ and $p<0.001$, respectively) than those for the BPD group. Meanwhile, both ATX and CA19-9 in TS1 \& TS2 were significantly higher $(p=0.005$ and $p<0.001$, respectively) compared to the HV group. However, LPA levels between the TS1 \& TS2 group and HVs did not differ significantly $(p=0.057)$. When the three biomarkers were segregated according to tumor stage, a significant elevation was seen for all three markers in patients with later PC stage (TS 3 \& TS4) compared to those with early PC stage (TS $1 \&$ TS2) $(p<0.001)$.

\section{Diagnostic evaluation of serum ATX and LPA in PC patients using CA19-9 as a standard marker}

The specificity and sensitivity of serum ATX and LPA levels were next assessed for PC diagnosis using CA19-9 as a standard diagnostic marker. The diagnostic efficacy of the three markers was assessed at $286 \mathrm{ng} / \mathrm{mL}, 10.7 \mu \mathrm{g} /$ $\mathrm{mL}$ and $57 \mathrm{U} / \mathrm{mL}$, which were selected as cut-off values for ATX, LPA and CA19-9, respectively (Fig. 1, Table 4). LPA had the highest sensitivity among all PC patients (91.74\%), and these values varied for late tumor stage (95.65\%) and early tumor stage $(85.67 \%)$ patients. Sensitivity was lower for ATX and CA19-9. CA19-9 had a sensitivity of $80.21 \%$ among all PC patients, and the sensitivity was $82.26 \%$ and $77.33 \%$ for late and early tumor stage, respectively. Meanwhile, ATX had a sensitivity of $78.95 \%$ for all PC patients, and for early and late tumor stage the values were $65.33 \%$ and $89.6 \%$, respectively. When tumor stage was disregarded, ATX was the most specific biomarker (80\%) followed by CA19-9 (75\%) and LPA (69.4\%) compared to healthy volunteers.

\section{Combining ATX and LPA with CA19-9 enhanced the diagnostic precision for early tumor stage PC}

We next attempted to determine whether combining ATX and LPA with CA19-9 improved the diagnostic accuracy for early stage PC. Compared with the HV group, the combination of ATX with CA19-9 enhanced the Areas Under the ROC Curves (AUROC) significantly $(p=0.0268)$, whereas adding the three biomarkers together further improved AUROC to $0.983 \pm 0.016$ $(p=0.0012)$. Furthermore, the combination of ATX with CA19-9 also enhanced the Areas Under the ROC Curve (AUROC) significantly $(p=0.0381)$ and adding all three biomarkers together further improved the AUROC to $0.973 \pm 0.023(p=0.0090)$ when compared with the BPD group (Table 5 and Fig. 2). Upon combining two or all three markers, the AUROC increased for the late tumor stage of PC, but the increase was not significant (CA19-9 and ATX vs. HV group, $p=0.4508$ ) (CA19-9 and ATX vs. BPD group, $p=0.279$ ) (ATX, LPA and CA19-9 vs.
HV group, $p=0.1657$ ) ( ATX, LPA and CA19-9 vs. BPD group, $p=0.136$ ) ( Table 5 and Fig. 2).

\section{Discussion}

Early diagnosis of PC can markedly increase the probability of successful radical surgery as well as improve disease outcome and prolong survival time [21]. Novel biomarkers or a suite of biomarkers are needed to allow early detection of PC.

Recently, a set of new markers derived from serum was recently assessed as PC markers [5]. Among them, increasing attention is being paid to the ATX-LPA signaling axis, which regulates a number of cellular activities [15]. In particular, levels of LPA are related to activated platelets that can both directly and indirectly promote tumor growth, metastasis, and immune evasion [22]. Moreover, LPA is reported to induce cell proliferation, migration, and invasion of gastric tumors such as PC [11]. The extracellular lysoPLD ATX catalyzes the conversion of LPC to LPA, and overexpression of ATX results in multiple physiological abnormalities [23]. As for PC, Nakai et al. [24] have found that among patients with various cancers of digestive system, increased serum ATX activity was predominantly observed among pancreatic cancer patients. Meanwhile, overexpression of both ATX and LPA in pancreatic tissues has been reported for PC patients and the ATX-LPA axis played a critical role and might be the potential target in pancreatic cancer [2527]. These findings suggest that measuring serum levels of ATX and LPA, either alone or in combination with the gold standard PC biomarker CA19-9 [4], could help distinguish patients with PC. In this study, we found that PC patients had significantly increased serum ATX and LPA levels, relative to HVs and BPD patients who had conditions such as benign biliary obstruction or chronic pancreatitis. The results also indicated that ATX and LPA levels may be affected by jaundice and the level of alkaline phosphatase. Furthermore, levels of all three biomarkers in samples from early tumor stage PC patients significantly differed from those seen for patients with late tumor stage. Together these results support the use of serum ATX and LPA as a diagnostic biomarker for PC.

An ideal tumor biomarker should have high sensitivity and high specificity. Biomarkers that have high sensitivity can detect related malignant tumors at an early stage, whereas biomarkers having high specificity are useful to make an accurate differential diagnosis [28]. In this study, CA19-9, when cut-off value was $57 \mathrm{U} /$ $\mathrm{mL}$, had $75 \%$ specificity and $80.21 \%$ sensitivity for the detection of PC among the entire study population, yet for detection of early PC (TS1 and 2) this sensitivity decreased to $77.33 \%$. Meanwhile, LPA, when cut-off value was $10.7 \mu \mathrm{g} / \mathrm{mL}$, had $91.74 \%$ sensitivity and $69.4 \%$ 

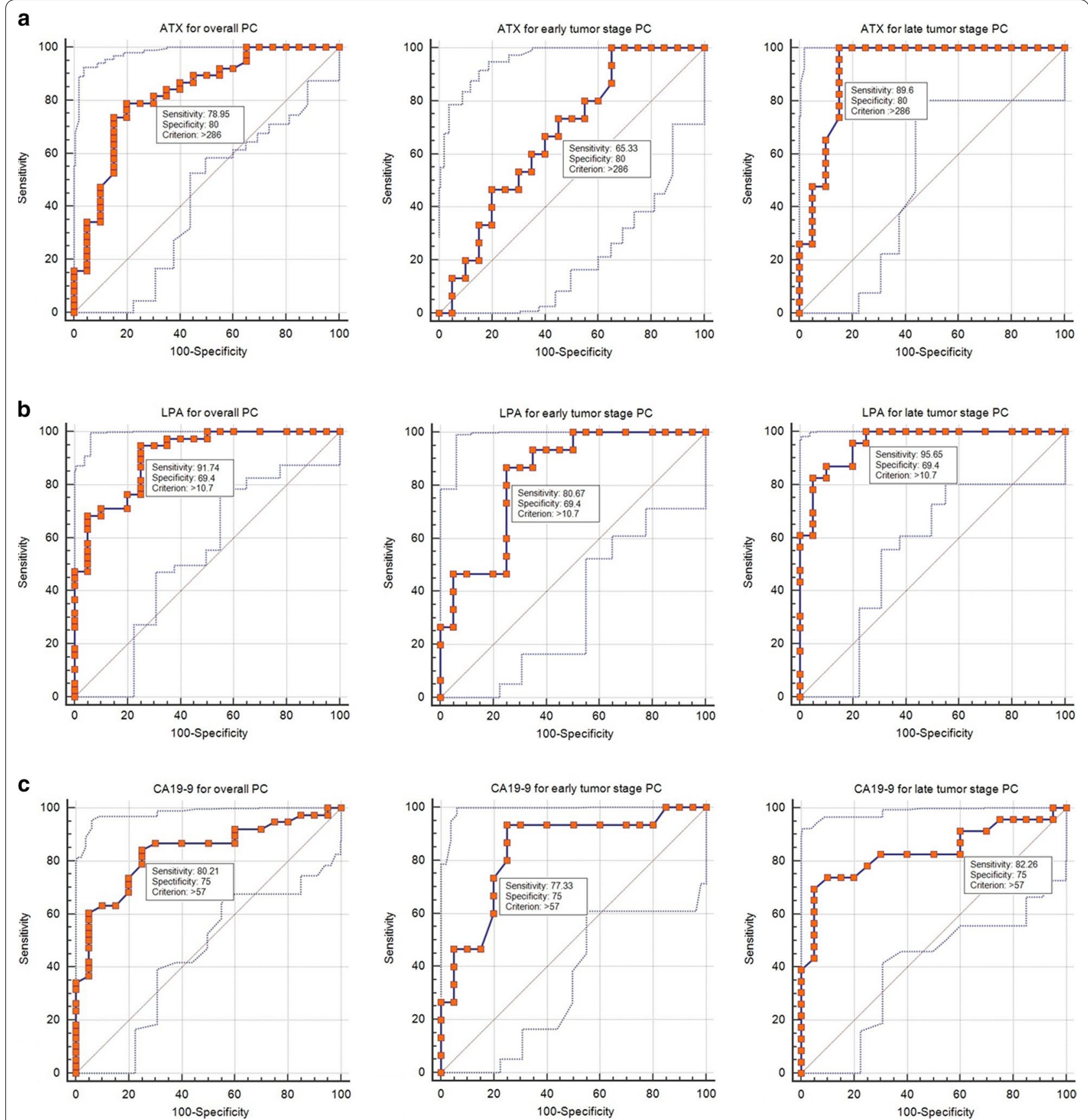

Fig. 1 Receiver Operating Characteristic (ROC) curve analysis of serum ATX, LPA, and CA19-9 levels for prediction of overall, early tumor stage, and late tumor stage PC relative to levels in BPD patients and HVs

specificity for detection of $\mathrm{PC}$ from among the total study population. Considering early stage PC patients, the sensitivity was $80.67 \%$. ATX, when cut-off value was $286 \mathrm{ng} / \mathrm{mL}$, had $80 \%$ specificity for early stage PC detection, but the sensitivity was $65.33 \%$. It was noteworthy that elevated serum ATX can be seen in tumors, autoimmune diseases, liver cirrhosis and other multiple system and organ diseases [15] and it might be related to the lower specificity of ATX compared with the gold standard. Therefore, strengthening the laboratory test of patients' related diseases and reducing the interference of related diseases or problems on serum ATX test might be able to enhance the specificity of ATX in PC 
Table 4 Sensitivity and specificity of potential diagnostic biomarkers in pancreatic cancer

\begin{tabular}{llll}
\hline Biomarkers & $\begin{array}{l}\text { Optimum } \\
\text { cut-off }\end{array}$ & Sensitivity (\%) & Specifificity (\%) \\
\hline PC vs. Controls & & & \\
CA19-9 & $\geq 57 \mathrm{U} / \mathrm{mL}$ & 80.21 & 75 \\
ATX & $\geq 286 \mathrm{ng} / \mathrm{mL}$ & 78.95 & 80 \\
LPA & $\geq 10.7 \mu \mathrm{g} / \mathrm{mL}$ & 91.74 & 69.4 \\
TS1 +TS2 vs. & & & \\
Controls & & & \\
CA19-9 & $\geq 57 \mathrm{U} / \mathrm{mL}$ & 77.33 & 75 \\
ATX & $\geq 286 \mathrm{ng} / \mathrm{mL}$ & 65.33 & 80 \\
LPA & $\geq 10.7 \mu \mathrm{g} / \mathrm{mL}$ & 80.67 & 69.4 \\
TS3+TS4 vs. & & & \\
Controls & $\geq 57 \mathrm{U} / \mathrm{mL}$ & 82.26 & 75 \\
CA19-9 & $\geq 286 \mathrm{ng} / \mathrm{mL}$ & 89.6 & 80 \\
ATX & $\geq 10.7 \mu \mathrm{m} / \mathrm{mL}$ & 95.65 & 69.4 \\
LPA &
\end{tabular}

Controls: healthy volunteers + benign pancreatic diseases

TS, tumor stage

Table 5 Potential combined ATX and/or LPA with CA19-9 biomarkers

\begin{tabular}{llll}
\hline Biomarkers & AUROC \pm SEM & $\mathbf{9 5 \% ~ C l}$ & $\boldsymbol{p}$ value \\
\hline TS1+TS2 vs. HVs & & & \\
CA19-9 & $0.837 \pm 0.0423$ & $0.673-0.939$ & \\
CA19-9+ATX & $0.953 \pm 0.0309$ & $0.823-0.996$ & $0.0268^{*}$ \\
CA19-9+ATX+LPA & $0.983 \pm 0.016$ & $0.970-0.999$ & $0.0012^{*}$ \\
TS1+TS2 vs. BPDs & & & \\
CA19-9 & $0.821 \pm 0.065$ & $0.758-0.972$ & \\
CA19-9+ATX & $0.926 \pm 0.041$ & $0.849-0.993$ & $0.0381^{*}$ \\
CA19-9+ATX+LPA & $0.973 \pm 0.023$ & $0.921-0.999$ & $0.0090^{*}$ \\
TS3+TS4 vs. HVs & & & \\
CA19-9 & $0.836 \pm 0.064$ & $0.691-0.931$ & \\
CA19-9+ATX & $0.898 \pm 0.0516$ & $0.767-0.969$ & 0.4508 \\
CA19-9+ATX+LPA & $0.991 \pm 0.0917$ & $0.902-0.999$ & 0.1657 \\
TS3+TS4 vs. BPDS & & & \\
CA19-9 & $0.835 \pm 0.094$ & $0.714-0.951$ & \\
CA19-9+ATX & $0.881 \pm 0.066$ & $0.827-0.992$ & 0.279 \\
CA19-9+ATX+LPA & $0.942 \pm 0.078$ & $0.913-0.999$ & 0.136 \\
\hline
\end{tabular}

HVs, healthy volunteers; BPDs, benign pancreatic diseases; TS, tumor stage; SEM, standard error of the mean; AUROC, Areas Under the ROC Curves

* Depicts significance at $p$ value $<0.05$

patients. LPA has better sensitivity but lower specificity, particularly for detection of late stage disease.

Detection of disease based on a single tumor biomarker can have certain limitations, whereas combined detection of multiple indicators can yield higher sensitivity and specificity, and thus would have important clinical

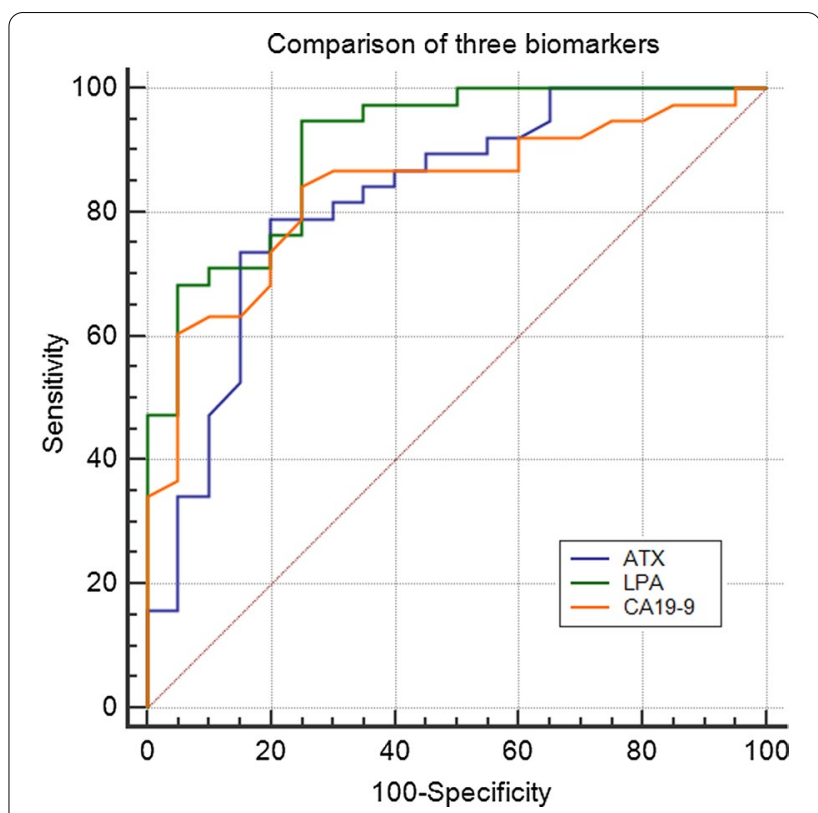

Fig. 2 Receiver Operating Characteristic (ROC) curve comparing ATX, $\angle P A$, and CA19-9 levels for early detection of PC relative to levels in BPD patients and HVs. The Areas Under the ROC Curve (AUROC) were 0.836 for CA19-9, 0.824, for ATX, and 0.908 for LPA

value for the early diagnosis of cancers such as PC [29]. In this study, we found that the AUROC for LPA alone or in combination with ATX or CA19-9 could significantly enhance its sensitivity for detection of early PC (TS1 and 2) relative to HVs and BPD patients, but this increase was not preserved for late stage (TS3 and 4) PC. Taken together, a combination of ATX, LPA, and CA19-9 as serum biomarkers may be a promising approach for early diagnosis of PC.

This study has several potential limitations. First, all data were compiled from patients treated at a single center. Inclusion of more diverse samples acquired from multiple centers is needed to verify the results of this study. Second, other biomarkers that have been used for early diagnosis of PC, such as CA125, CA242 and CA50, were not considered in this study. Third, this study did not include patients having other periampullary tumors such as distal cholangiocarcinomas, pancreatic neuroendocrine tumors and ampullary/duodenal cancers. Last, a large-scale study to examine tumor biomarker combinations and evaluate strategies for early diagnosis of PC is needed.

\section{Conclusion}

This study performed a quantitative survey of the diagnostic potential of three biomarkers ATX, LPA and CA19-9 for the early detection of PC. Compared with HVs, the serum levels of all three biomarkers increased 
substantially in samples from patients with early stage PC. Moreover, the AUROC and diagnostic efficiency was enhanced significantly by the addition of ATX and LPA to CA19-9. These results showed that combined application of LPA and ATX with CA19-9 can be used for early detection of PC.

\section{Abbreviations}

PC: Pancreatic cancer; ATX: Autotaxin; LPA: Lysophosphatidic acidhealthy volunteers (HVs; BPDs: Benign pancreatic diseaseslysophospholipase D (lysoPLD)-; LPC: Lysophosphatidylcholine; ENPP2: Ectonucleotide pyrophosphatase/ phosphodiesterase 2receiver; ROC: Operating characteristics; AUROC: Areas Under the ROC Curve.

\section{Acknowledgments}

Not applicable.

\section{Authors' contributions}

JC conceived and drafted the manuscript. WDX and HYL performed data extraction and statistical analyses; XZG gave critical comments and revised the manuscript. All authors have read and approved the final manuscript, and ensured that this was the case.

\section{Funding}

This work was supported by the National Natural Science Foundation of China (no. 81672465). The funding body gave critical comments in the design of the study and collection, analysis, and interpretation of data and revised the manuscript in writing the manuscript.

\section{Availability of data and materials}

The data that support the findings of this study are available from the general hospital of northern theater command but restrictions apply to the availability of these data, which were used under license for the current study, and so are not publicly available. Data are however available from the authors upon reasonable request and with permission of the general hospital of northern theater command.

\section{Ethics approval and consent to participate}

The research protocol was approved by the Ethics Committee of the General Hospital of Northern Theater Command (approval no. K(2011)49). Informed written consent was obtained for all participants.

\section{Consent to publish}

Not applicable.

\section{Competing interests}

There were no any financial and non-financial competing interests in this article.

\section{Received: 2 August 2019 Accepted: 2 February 2021}

Published online: 10 February 2021

\section{References}

1. Wolfgang $C L$, Herman JM, Laheru DA, Klein AP, Erdek MA, Fishman EK, Hruban $\mathrm{RH}$. Recent progress in pancreatic cancer. CA Cancer J Clin. 2013:63:318-48.

2. Hidalgo M. Pancreatic cancer. N Engl J Med. 2010;362:1605-17.

3. Poruk KE, Firpo MA, Adler DG, Mulvihill SJ. Screening for pancreatic cancer: why, how, and who? Ann Surg. 2012;257(1):17-26.

4. Duraker N, Hot S, Polat Y, Hobek A, Gencler N, Urhan N. CEA, CA 19-9, and CA125 in the differential diagnosis of benign and malignant pancreatic diseases with or without jaundice. J Surg Oncol. 2007;95(2):142-7.

5. Harsha HC, Kandasamy K, Ranganathan P, Rani S, Ramabadran S, Gollapudi S, Balakrishnan L, Dwivedi SB, Telikicherla D, Selvan LD,
Goel R, Mathivanan S, Marimuthu A, Kashyap M, Vizza RF, Mayer RJ, Decaprio JA, Srivastava S, Hanash SM, Hruban RH, Pandey A. A compendium of potential biomarkers of pancreatic cancer. PLoS Med. 2009;6(4):e1000046.

6. Grantzdorffer I, Carl-McGrath S, Ebert MP, Rocken C. Proteomics of pancreaticcancer. Pancreas. 2008;36(4):329-36.

7. Bandoh K, Aoki J, Hosono H, Kobayashi S, Kobayashi T, MurakamiMurofushi K, Tsujimoto M, Arai H, Inoue K. Molecular cloning and characterization of a novel human G-protein-coupled receptor, EDG7, for lysophosphatidic acid. J Biol Chem. 1999;274(39):27776-85.

8. Willier S, Butt E, Grunewald TG. Lysophosphatidic acid (LPA) signalling in cell migration and cancer invasion: a focussed review and analysis of LPA receptor gene expression on the basis of more than 1700 cancer microarrays. Biol Cell. 2013;105:317-33.

9. Choi JW, Herr DR, Noguchi K, Yung YC, Lee CW, Mutoh T, Lin ME, Teo ST, Park KE, Mosley AN, Chun J. LPA receptors: subtypes and biological actions. Annu Rev Pharmacol Toxicol. 2010;50:157-86.

10. Aoki J. Mechanisms of lysophosphatidic acid production. Semin Cell Dev Biol. 2004;15:477-89.

11. Ramachandran S, Shida D, Nagahashi M, Fang X, Milstien S, Takabe K, Spiegel S. Lysophosphatidic acid stimulates gastric cancer cell proliferation via ERK1-dependent upregulation of sphingosine kinase 1 transcription. FEBS Lett. 2010;584:4077-82.

12. Stracke ML, Krutzsch HC, Unsworth EJ, Arestad A, Cioce V, Schiffmann E, Liotta LA. Identification, purification, and partial sequence analysis of autotaxin, a novel motility-stimulatingprotein. J Biol Chem. 1992:267(4):2524-2519.

13. Goding JW, Grobben B, Slegers H. Physiological and pathophysiological functions of the ecto-nucleotide pyrophosphatase/phosphodiesterase family. Biochim Biophys Acta. 2003;1638(1):1-19.

14. Umezu-Goto M, Kishi Y, Taira A, Hama K, Dohmae N, Takio K, Yamori T, Mills GB, Inoue K, Aoki J, Arai H. Autotaxin has lysophospholipase D activity leading to tumor cell growth and motility by lysophosphatidic acid production. J Cell Biol. 2002;158(2):227-33.

15. Kazama S, Kitayama J, Aoki J, Mori K, Nagawa H. Immunohistochemical detection of autotaxin (ATX)/lysophospholipase D (lysoPLD) in submucosal invasive colorectal cancer. J Gastrointest Cancer. 2011;42:204-11.

16. Leblanc $R$, Peyruchaud $O$. New insights into the autotaxin/LPA axis in cancer development and metastasis. Exp Cell Res. 2015;333:183-9.

17. Peyruchaud $O$, Saier L, Leblanc R. Autotaxin implication in cancer metastasis and autoimunne disorders: functional implication of binding autotaxin to the cell surface. Cancers. 2020;12:105.

18. Tang X, Benesch K, Brindley N. Role of the autotaxin-lysophosphatidate axis in the development of resistance to cancer therapy. Biochim Biophys Acta Mol Cell Biol Lipids. 2020;1865:158716.

19. Nishigaki D, Yamamoto R, Shinzawa M. Body mass index modifies the association between frequency of alcohol consumption and incidence of hypertension in men but not in women: a retrospective cohort study. Hypertens Res. 2020;43:322-30.

20. Stuart A, Singh G, Ramos A. Relationship of pancreas volume to tobacco smoking and alcohol consumption following pancreatitis. Pancreatology. 2020;20(1):60-7.

21. Ballehaninna UK, Chamberlain RS. Biomarkers for pancreatic cancer: promising new markers and options beyond CA 19-9. Tumour Biol. 2013:34(6):3279-92.

22. Honn KV, Tang DG, Chen YQ. Platelet cancer interactions. Semin Thromb Hemost. 2014:40:296-305.

23. Perrakis A, Moolenaar WH. Autotaxin: structure-function and signaling. J Lipid Res. 2014;6:1010-8.

24. Nakai $Y$, Ikeda $H$, Nakamura K. Specific increase in serum autotaxin activity in patients with pancreatic cancer. Clin Biochem. 2011:44(8-9):576-81.

25. Fukushima K, Takahashi K, Yamasaki E, Onishi Y, Fukushima N, Honoki $\mathrm{K}$, Tsujiuchi T. Lysophosphatidic acid signaling via LPA1 and LPA3 regulates cellular functions during tumor progression in pancreatic cancer cells. Exp Cell Res. 2017:352(1):139-45.

26. Quan M, Cui J, Feng $X$. The critical role and potential target of the autotaxin/lysophosphatidate axis in pancreatic cancer. Tumor Biology. 2017:39(3):1010428317694544. 
27. Auciello FR, Bulusu V, Oon C. A stromal lysolipid-autotaxin signaling axis promotes pancreatic tumor progression. Cancer Discov. 2019;9(5):617-27.

28. Banfi G, Bravi S, Ardemagni A, Zerbi A. CA 199, CA 242 and CEA in the diagnosis and follow-up of pancreatic cancer. Int J Biol Markers. 2006;11(2):77-81.

29. Liu F, Du F, Chen X. Multiple tumor marker protein chip detection system in diagnosis of pancreatic cancer. World J Surg Oncol. 2014;12:333.

\section{Publisher's Note}

Springer Nature remains neutral with regard to jurisdictional claims in published maps and institutional affiliations.
Ready to submit your research? Choose BMC and benefit from:

- fast, convenient online submission

- thorough peer review by experienced researchers in your field

- rapid publication on acceptance

- support for research data, including large and complex data types

- gold Open Access which fosters wider collaboration and increased citations

- maximum visibility for your research: over 100M website views per year

At BMC, research is always in progress.

Learn more biomedcentral.com/submissions 